\title{
SKEW COMPENSATION IN ALL OPTICAL BIT PARALLEL WDM SYSTEMS
}

\author{
M. E. Vieira Segatto* , F. N. Timofeev, R. Wyatt, R. Kashyap ${ }^{\dagger}$ \\ Corning Research Centre \\ Adastral Park, Martlesham Heath, Ipswich \\ IP5 3RE, United Kingdom. \\ segatto@ele.ufes.br
}

J. R. Taylor

Femtosecond Optics Group, The Blackett Labs. Imperial College, London $S W^{7}$ 2BZ, United Kingdom.

jr.taylor@ic.ac.uk

\begin{abstract}
A bit skew and dispersion compensation scheme using fibre gratings for a $10 \mathrm{~Gb} / \mathrm{s}$-channel, 2-bit, Bit Parallel WDM system over $100 \mathrm{~km}$ of standard single-mode fibre is presented. The combination of channelised dispersion, and additional fibre delay, is sufficient to give full compensation.
\end{abstract}

Keywords: Optical Parallel Transmission, Bit Skew Compensation

\section{Introduction}

High-speed digital transmission between two points can be implemented basically in three different ways as illustrated in figure 1 . On the serial system (figure 1(a)), a Serialiser is used to convert the bits of a digital word in a stream of bits. This stream is then used to modulate an optical beam and sent through the fibre to the receiver. At the receiver side, the optical signal is converted to electrical pulses and a deserialiser generates the original binary word. As the bits are transmitted serially, to form a word the time equivalent of a number of bits in a word at

\footnotetext{
* Federal University of Espírito Santo, Brazil. Also on leave of absence at Imperial College. This work was partially supported by CAPES/Brazil.

t Raman Kashyap is now with Corvis Canada.
}

The original version of this chapter was revised: The copyright line was incorrect. This has been corrected. The Erratum to this chapter is available at DOI: 10.1007/978-0-387-35491-0_28 
the bit period plus the time for serial-to-parallel and parallel-to-serial conversions is required. Figure 1(b) shows an alternative to the serial transmission system. In order to avoid the serial-parallel-serial conversions, each bit is sent using a different fibre. The Bit Parallel WDM (BP-WDM) shown in figure 1(c) uses WDM technology to transmit information. Each bit is used to modulate the optical source at a different wavelength. The wavelengths are then multiplexed and launched into the fibre. After the transmission, the wavelengths are separated and the digital word is recovered. Besides the well-known impairments in the serial system such as attenuation, dispersion and non-linearities, the parallel system is also quite sensitive to the propagation delay differences among the bits of a digital word. For both parallel systems, Figures 1(b) and $1(\mathrm{c})$, each bit travels in different optical paths. While in the ribbon fibre system the refractive index and length differences are responsible for most of the bit-skew, for the BP-WDM system it is caused by the fibre dispersion characteristic. Practical systems using ribbon fibres are restricted to short distances and low-speed due to high values of bit-skew having its use restricted to boards and inter-frame connections and to short-distance links $[1,2,3]$. For high performance computer interconnections, for instance, the BP-WDM system can be a good option since it is able to cover higher bit-rates and distances by using just one fibre $[4,5,6]$.

In this paper we present results on a two-channel (two-bit) BP-WDM system operating at $10 \mathrm{~Gb} / \mathrm{s}$ per channel over $100-\mathrm{km}$ of Standard Single Mode (SSM) fibre. The bit skew and dispersion were compensated using two apodised step Chirped Fibre Gratings (CFG). Section 1 presents the basic ideas of BP-WDM systems. Bit skew and bit skew compensation are discussed in sections 2 and 3. Experimental results for the bit skew compensator is presented in section 4.

\section{BP-WDM TRANSMISSION}

In 1988, Loeb and Stilwell [7] proposed the fist long-distance parallel system using WDM technology. In his system, each bit of a digital word is transmitted on a separated wavelength, as illustrated in figure 2. This Bit Parallel WDM system uses a single fibre, eliminating the limitations imposed by multiple optical path lengths. Bit-skew remains a problem in a long-distance optical link operating in the first and third transmission windows where a standard fibre exhibits large group delay dispersion.

Three major questions must be answered when designing a BP-WDM system. The first question is in which transmission window the system should work. VCSEL and photodiode arrays operating in $850 \mathrm{~nm}$ 


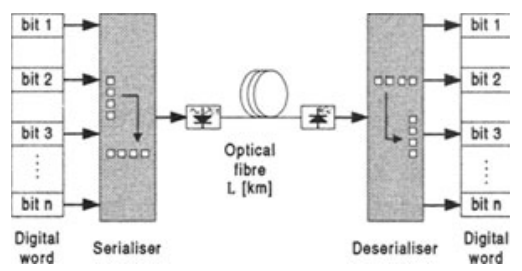

(a)

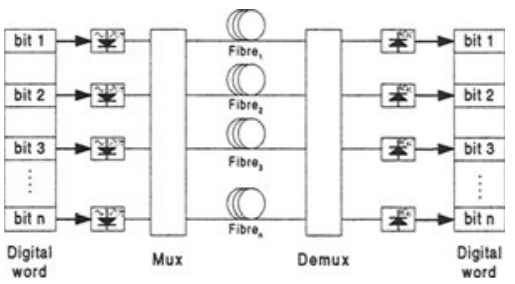

(b)

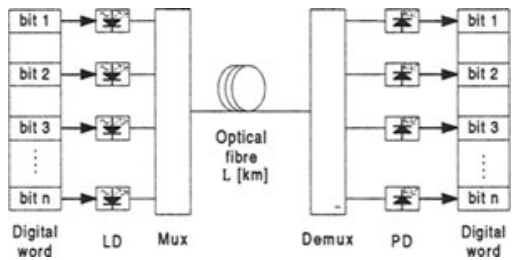

(c)

Figure 1 High-speed digital systems. (a) Serial system, (b) ribbon fibre system, and (c) parallel WDM system. LD:laser diode, PD: Photodetector.

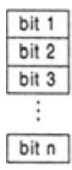

igital word

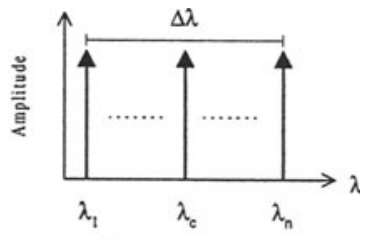

Wavelength Spectrum

Figure 2 Wavelength allocation_in BP-WDM systems. 
wavelength window have low manufacturing costs but intramodal and intermodal dispersion are very high for fibres at this wavelength window and link length would be severely limited. Single mode fibres operating in the second window have low dispersion but relatively higher losses if compared with the losses in the third window. On the other hand, the low losses and the availability of optical amplifiers in the third window favour long-distance transmission. Another factor is that WDM systems are available commercially mostly in the third window $[8,9]$.

The second question is the rate at which each bit-channel should operate. The main aim of a serial WDM transmission is to fully utilise the potential throughput of an optical fibre, by keeping the data bit rate for individual wavelength channels as high as possible. Instead, a BP-WDM system endeavours to reduce the speed per bit-channel by the number of optical channels, thus simplifying implementation of the required signal processing in optical networks. Although paralleling the data path increases the number of electronic circuits by a factor equal to the number of channels, lowered speed translates into lower prices for the electronic systems. For the experiments shown in this paper, the rate per bitchannel chosen was $10 \mathrm{Gbit} / \mathrm{s}$ for two reasons. From the electronic point of view the performance of computers and the associated integrated circuits increases by a factor of two every 18 months [10] which means that in few years computer clocks at 5 or $10 \mathrm{GHz}$ will be part of our reality. From the optical point of view, the effects of dispersion in the system performance are apparent for a few kilometres of fibre and can also be studied. The third question is link length. Bit skew and dispersion are the import factors to answer this question. The maximum link length is mainly defined by the fibre dispersion and any attempt to increase it will depend on bit skew compensation techniques.

Bit skew compensation techniques can be divided into two categories, electronic compensation [11] and all optical compensation [12]. Electronic compensation implies more complicated transmitter and receiver systems and consequently in more expensive systems. However, all optical compensation can be used without increasing the complexity of the system.

\section{BIT SKEW}

The bit skew is the major problem in BP-WDM systems. It can be caused by several factors such as turn-on delay in semiconductor lasers, dispersion in optical devices such as filters and (de)multiplexer, and mainly by the group delay dispersion of the optical fibre [12]. 
As illustrated in figure 2, a digital word with $n$ bits is mapped into the optical domain using one wavelength per bit. As each bit is represented by a different wavelength, they suffer different group delay due to the dispersion characteristics of the fibre and arrive at the end of the fibre at different times.

The bit skew in the fibre can be written as a function of the dispersion as follow: The total dispersion in a single mode fibre, expressed as function of the dispersion slope $S_{0}$, the zero-dispersion wavelength $\lambda_{0}$ and wavelength $\lambda$, is given by the Sellmeier expression [13]

$$
D(\lambda)=\frac{S_{0} \lambda}{4}\left[1-\left(\frac{\lambda_{0}}{\lambda}\right)^{4}\right] .
$$

The maximum bit skew can be determined by the difference in the group delay between the fastest and the slowest channel. For the sake of simplicity, we assume that all channels are on the same side of the zero-dispersion wavelength. Then, the delay between the first channel and the last channel gives the maximum bit skew.

If we define a system with $n$ channels (i.e. a digital word with $n$ bits) equally spaced and total bandwidth $\Delta \lambda$, as shown in figure 2 , the wavelength of the first channel and of the last channel can be expressed as a function of the central wavelength $\lambda_{c}$ as

$$
\begin{aligned}
& \lambda_{1}=\lambda_{c}-\Delta \lambda / 2, \\
& \lambda_{n}=\lambda_{c}+\Delta \lambda / 2 .
\end{aligned}
$$

The group delay $\tau_{g}$ in a fibre of length $L$ is determined by integrating the total dispersion over the wavelength, and

$$
\frac{\tau_{g}}{L}=\int D\left(\lambda^{\prime}\right) d \lambda^{\prime} .
$$

The maximum bit skew per unit of length is given by

$$
\frac{\Delta \tau_{g}}{L}=\int_{\lambda_{1}}^{\lambda_{n}} D\left(\lambda^{\prime}\right) d \lambda^{\prime} .
$$

Substituting Eq. (1) in (4) and using (2), we have

$$
\frac{\Delta \tau_{g}}{L}=\Delta \lambda \frac{\lambda_{c} S_{0}}{4}\left[1-\frac{\lambda_{0}^{4}}{\left(\lambda_{c}^{2}-\Delta \lambda^{2} / 4\right)^{2}}\right] .
$$




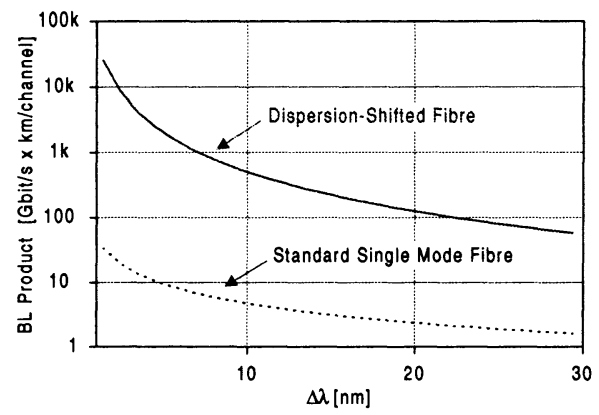

Figure 3 The bit rate-distance product as a function of the total bandwidth for standard single mode and dispersion-shifted fibres when the maximum bit skew allowed is equal to one bit period.

Assuming that $\lambda_{c} \gg \Delta \lambda$, then

$$
\frac{\Delta \tau_{g}}{L} \cong \Delta \lambda \frac{\lambda_{c} S_{0}}{4}\left[1-\left(\frac{\lambda_{0}}{\lambda_{c}}\right)^{4}\right]=\Delta \lambda D\left(\lambda_{c}\right)
$$

Equation (6) shows that the maximum bit skew is a function of the total bandwidth and of the dispersion of the fibre at the central wavelength. The only assumptions made here are that the central wavelength must be much greater than the total bandwidth and all wavelengths are in the same side of the fibre dispersion curve. This expression holds even if different expressions are used for the dispersion equation (1).

The bit rate-distance (BL) product can be easily computed from (6) if a maximum bit skew is defined. Figure 3 shows the BL product per channel for standard single mode fibre and for dispersion-shifted (DS) fibre $^{1}$ when the central wavelength is $1538 \mathrm{~nm}$ and the maximum bit skew allowed is equal to one bit period. High products can be obtained for DS fibres [4] if the spacing between wavelengths is kept small.

\section{BIT SKEW COMPENSATION}

A number of techniques have been proposed to deal with bit skew. These techniques include the use of dispersion-shifted fibre [4], electronic bit realignment in the receiver [11], and a shepherd pulse technique [14] based on nonlinear optics. Drawbacks of these techniques include lack of resynchronisation accuracy ( $100 \mathrm{ps})$ or high pulse energy to induce fibre nonlinearities. In addition, the shepherd pulse technique requires that the passive component skew be within the capture range of the 


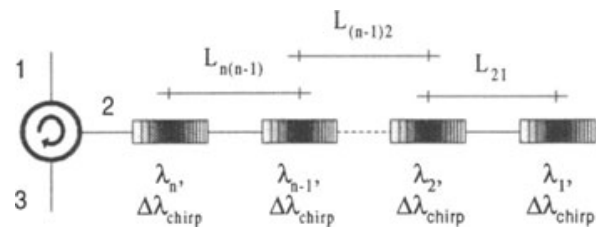

Figure 4 The fibre grating configuration used for bit skew and dispersion compensation.

shepherd pulse ( $\sim 5 \mathrm{ps})$, which complicates the design of optical couplers and demultiplexers [9].

In this paper, we propose the use of of step Chirped Fibre Gratings (CFG) to simultaneously compensate bit skew and dispersion in 10 Gbit/s-channel parallel WDM systems. The system works as follows: The group delay in Standard Single Mode fibres (SSM) increases with the wavelength in the anomalous-dispersion regime. Bits travelling in lower wavelengths will arrive at the receiver end before those in higher wavelengths, so additional delay must be added to the faster bits.

Figure 4 shows the Fibre Grating Bit Skew Compensator (FGBSC). It consists of a three port circulator and a set of fibre gratings. Light coming from the fibre enters into the circulator and is reflected by the set of gratings. Each wavelength is reflected in a different position of the set of gratings, so the delay imposed by the FGBSC can be used to compensate to the fibre skew.

Chirped gratings have been extensively used as dispersion compensation devices [15] due their compactness and dispersion characteristics. Each grating acts as a selective mirror, reflecting one wavelength. The gratings are sliced in such way that long wavelengths are reflected first and the device delay is defined by the separation of the gratings. Some degree of tuning is possible by mechanically stressing the gratings. The two mechanisms, the separation of the gratings and the chirp are responsible to compensate to the fibre dispersion and completely eliminate the bit skew.

\section{EXPERIMENTAL RESULTS}

The experimental setup used to measure bit skew and dispersion is shown in Figure 5. The optical transmitter includes two semiconductor lasers with external fibre grating [16], directly modulated at $10 \mathrm{~Gb} / \mathrm{s}$ at wavelengths $1554 \mathrm{~nm}$ and $1556.8 \mathrm{~nm}$. After combining, the two wavelengths optical power $\sim 3 \mathrm{dBm}$ per channel was launched into $50-\mathrm{km}$ of 
SSM fibre, optically amplified and launched again into a further $50-\mathrm{km}$ of SSM fibre. Two 10-cm long step-chirped gratings [15, 17] with reflection bandwidth $0.42 \mathrm{~nm}$ and $0.48 \mathrm{~nm}$, respectively, separated by a short length of SSM are used to compensate the chromatic dispersion in each channel, and the overall bit skew. The per-channel dispersion of around $1200 \mathrm{ps} / \mathrm{nm}$, corresponding to about $72 \mathrm{~km}$ of SSM fibre is compensated by the grating chirp, while the overall bit skew is compensated by the optical separation between the centres of the gratings $(\Delta l)$. Figure 6 shows the transmitted and received optical spectra. A fibre grating operating in reflection in conjunction with a circulator provides $\sim 1 \mathrm{~dB}$ insertion loss for both channels. Very small grating reflectivity outside the reflection band provides good filtering of optical ASE noise from inline optical amplifier. Both gratings were fine-tuned using strain to the wavelength of the corresponding channel. Eye diagrams measured for channel 1 both back to back and after fibre + compensator are shown in Figure 7(a) and 7(b), confirming good reshaping of signal from directly modulated laser sources. The transmitted and received waveforms for both channels are shown in Figure 8, demonstrating total bit skew compensation. The bit skew after $100 \mathrm{~km}$ of fibre was around $2.5 \mathrm{~ns}$. The propagation time delay compensation has been done using $\Delta l \sim 9 \mathrm{~cm}$ of fibre spliced between the two gratings.

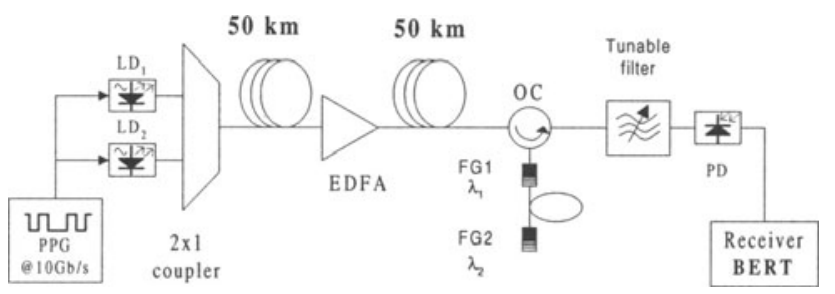

Figure 5 Experimental setup.

\section{CONCLUSIONS}

In this paper, we have proposed a new scheme for all optical bit skew compensation in bit parallel WDM systems. We have demonstrated 2 bits BP-WDM transmission, $10 \mathrm{~Gb} / \mathrm{s}$ per channel, over $100 \mathrm{~km}$ of SSM fibre using directly modulated lasers with external fibre gratings. Simultaneous chromatic dispersion (-1200 ps/nm) and bit skew ( $2.5 \mathrm{~ns}$ ) compensation were achieved. We believe that systems using BP-WDM will be useful for computer interconnects in high-speed parallel systems and ring networks. 


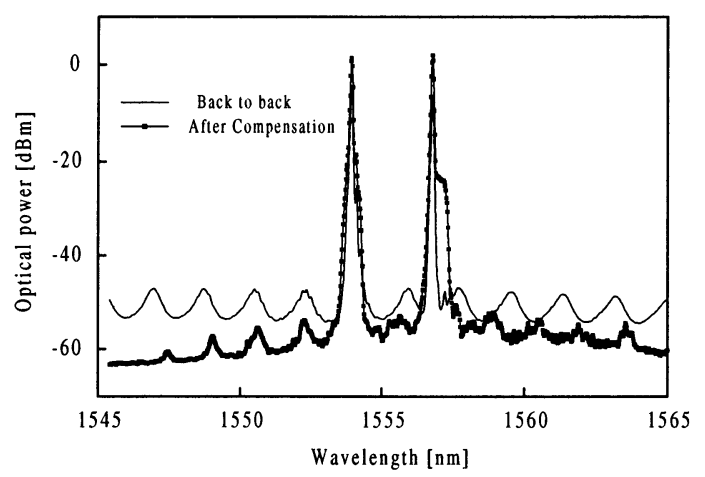

Figure 6 Transmitted and received optical spectra.

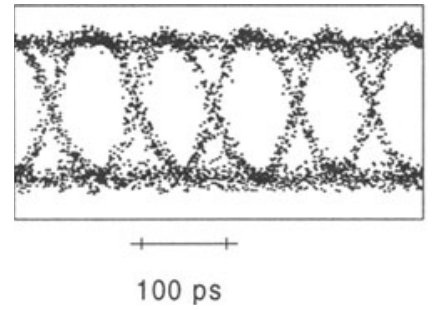

(a)

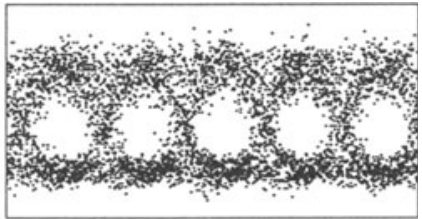

(b)

Figure 7 (a) Transmitted and (b) received eye diagrams.

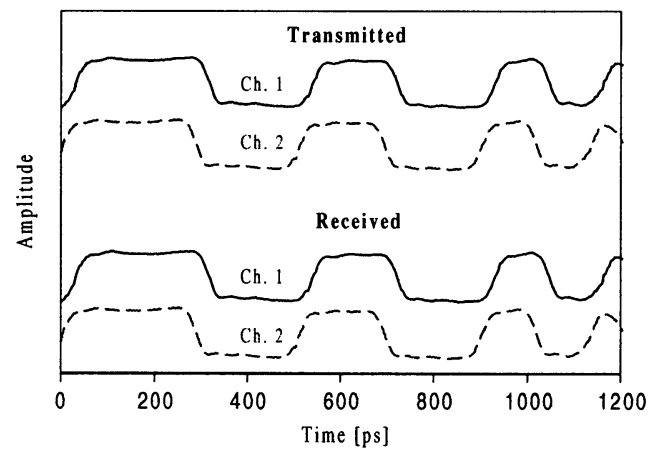

Figure 8 Transmitted and received waveforms for both channels. 


\section{References}

[1] N. Fujimoto, K. Wakao, and M. Yano. Intelligent parallel optical link - parallel optical transmission system and LSIs. FUJITSU Sci. Tech. J., 30(2):214-23, December 1994.

[2] A. Takai, T. Kato, S. Yamashita, S. Hanatani, Y. Motegi, K. Ito, $\mathrm{H}$. Abe, and H. Kodera. 200-Mb/s/ch 100-m optical subsystem interconnections using 8-channel $1.3-\mu \mathrm{m}$ laser diode arrays and singlemode fiber arrays. IEEE J. Lightwave Tech., 12(2):260-270, February 1994.

[3] H. Westphal and J. M. Spaus. Interfacing parallel computers to fiber-optic communication links for plant automation. In Proc. IEEE Electronic Components and Technology Conference, pages 805-810, 1994.

[4] L. Bergman, J. Morookian, and C. Yeh. An all-optical long-distance multi-Gbytes/s bit-parallel WDM single-mode fiber link. IEEE J. Lightwave Technol., 16:1577-1582, September 1998.

[5] K. M. Sivalingam. A comparison of bit-parallel and bit-serial architectures for WDM networks. Journal on Photonic Network Communications, 1:1-27, June 1999.

[6] L. Kleinrock et. al. The supercomputer supernet testbed: A WDMbased supercomputer interconnect. IEEE J. Lightwave Technol., 14(6):1388-1399, June 1996.

[7] M. L. Loeb and G. R. Stilwell Jr. High-speed data transmission on an optical fiber using a byte-wide WDM system. IEEE J. Lightwave Technol., 6:1306-1311, August 1988.

[8] L. Bergman, J. Morookian, and C. Yeh. WDM component requirements for bit-parallel fiber optic computer networks. In Proc. SPIE, pages 2-13, 1998 . 
[9] A. J. Mendez. Design of constant optical path couplers for bitparallel WDM systems. In Proc. SPIE, volume 3234, pages 94-99, 1998.

[10] A. F. J. Levi. Optical interconnects in systems. Proceedings of IEEE, 88(6):750-757, June 2000.

[11] M. L. Loeb and G. R. Stilwell Jr. An algorithm for bit-skew correction in byte-wide WDM optical fiber systems. IEEE J. Lightwave Technol, 8(2):239-242, February 1990.

[12] M. E. Vieira Segatto, R. Kashyap, G. D. Maxwell, J. R. Taylor, V.A. Bhagavatula, G.E. Berkey, and A.F. Evans. Multi Gb/s bit parallel WDM transmission using dispersion managed fibers. IEEE Photonics Tech. Letters, 12(8):925-927, August 2000.

[13] J. M. Senior. Optical Fiber Communications: Principles and Practice. Prentice Hall, London, 1992.

[14] L. Bergman and C. Yeh. Experimental verification of the pulse shepherding concept in dispersion-shifted single mode fiber for bitparallel wavelength links. In Proc. Fourth International Conference on Massively Parallel Processing Using Optical Interconnections (MPPOI '97), pages 25-29, Montreal-Canada, June 1997.

[15] R. Kashyap. Fibre Bragg Gratings. Academic Press, San Diego, 1999.

[16] F. N. Timofeev, I. A. Kostko, P. Bayvel, O. Berger, R. Wyatt, R. Kashyap, I. F. Lealman, and G. D. Maxwell. $10 \mathrm{Gbit} / \mathrm{s} \mathrm{di-}$ rectly modulated, high temperature-stability external fibre grating laser for dense WDM networks. Electron. Letters, 35(20):1737-1739, September 1999.

[17] I. Riant, S. Gurib, J. Gourhant, P. Sansonetti, C. Bungarzeanu, and R. Kashyap. Chirped fiber gratings for WDM chromatic dispersion compensation in multispan 10-Gb/s transmission. IEEE Journal of Selected Topics in Quantum Electronics, 5(5):1312-1324, September 1999. 